\title{
HOMOLOGY IN VARIETIES OF GROUPS. III
}

\author{
BY \\ C. R. LEEDHAM-GREEN
}

\begin{abstract}
A spectral sequence is used to calculate approximately the homology groups $\mathfrak{B}_{2}(\Pi, Z)$ as defined in the first paper in this series, for $\Pi$ a finitely generated abelian group and $\mathfrak{B}$ the variety of all nilpotent groups of class at most $c$.
\end{abstract}

Introduction. In previous papers [21] and [25], henceforth referred to as [HI] and [HII] respectively, (co-) homology groups $\mathfrak{B}_{n}(\Pi, A), \mathfrak{B}^{n}(\Pi, A)$ were discussed, where $\mathfrak{B}$ is a variety containing $\Pi$, and $A$ is a suitable $\Pi$-module. If $\mathfrak{B}$ is a variety containing $\mathfrak{B}$, there are homomorphisms

$$
\phi_{n}: \mathfrak{W}_{n}(\Pi, A) \rightarrow \mathfrak{B}_{n}(\Pi, A) \quad \text { and } \quad \phi^{n}: \mathfrak{B}^{n}(\Pi, A) \rightarrow \mathfrak{W}^{n}(\Pi, A) .
$$

Their basic properties are discussed in $\S 1$, and a spectral sequence with $\phi_{n}$ as an edge homomorphism is constructed in $\$ 2$. Similar spectral sequences have been constructed by various authors; the point of this treatment is to calculate the edge homomorphisms. Using the exact sequence of terms of low degree, the wild behaviour of $\mathfrak{B}_{2}(\Pi, Z)$ is demonstrated. In so far as one's intuition is based on the homology of groups, this comes near to being a universal counterexample.

The conventions and definitions used in [HI] and [HII] will remain in force. In particular the reader is referred to [HI, §1] for the definition of the (co-) homology groups $\mathfrak{B}_{n}(\Pi, A)$ and $\mathfrak{B}^{n}(\Pi, A)$.

1. Change of variety morphisms. If $P_{*}^{\mathfrak{B}} \rightarrow \Pi$ and $P_{*}^{\mathfrak{W}} \rightarrow \Pi$ are simplicial resolutions of $\Pi$ by $\mathfrak{B}$-splitting groups and $\mathfrak{W}$-splitting groups respectively, then since $\mathfrak{W}$ contains $\mathfrak{B}$ there is a simplicial map of $P_{*}^{\mathfrak{M}}$ into $P_{*}^{\mathfrak{B}}$ over $1_{\Pi}$ which is unique up to homotopy, (cf. Tierney and Vogel [18]). For example if $\Pi B_{*}^{\mathfrak{P}} \rightarrow \Pi$ and $\Pi B_{*}^{\mathfrak{P}} \rightarrow \Pi$ are the Barr-Beck resolutions of $\Pi$ in $\mathfrak{B}$ and $\mathfrak{W}$ respectively (see [HI, §2]), so that $\Pi B_{n}^{\mathfrak{B}}$ is $\mathfrak{B}$-free on $\Pi B_{n-1}^{\mathfrak{B}}, n \geqq 0, \Pi B_{-1}^{\mathfrak{Q}}=\Pi$, and $\Pi B_{n}^{\mathfrak{B}}$ is similarly defined, then a simplicial map $\eta_{*}: \Pi B_{*}^{\mathfrak{P}} \rightarrow \Pi B_{*}^{\mathfrak{Q}}$ may be defined inductively by $[w] \eta_{n}=w \eta_{n-1}$, where $w \in \Pi B_{n-1}^{\mathfrak{M}},[w]$ is the corresponding $\mathfrak{W}$-free generator of $\Pi B_{n}^{\mathfrak{M}}$, and $\left[w \eta_{n-1}\right]$ is the $\mathfrak{B}$-free generator of $\Pi B_{n}^{\mathfrak{Q}}$ corresponding to $w \eta_{n-1} ; \eta_{-1}=1_{\Pi}$. If $A$ is a left $\mathfrak{B} \Pi$-module, using these simplicial resolutions to calculate $\mathfrak{B}_{*}(\Pi, A)$ and $\mathfrak{B}_{*}(\Pi, A)$, one obtains well-defined homomorphisms

$$
\phi_{n}(\mathfrak{W}, \mathfrak{B}, \Pi, A): \mathfrak{W}_{n}(\Pi, A) \rightarrow \mathfrak{B}_{n}(\Pi, A), \quad n \geqq 0 .
$$

Received by the editors September 28, 1970.

AMS 1970 subject classifications. Primary 18H40, 20E10; Secondary 18C15, 18G10, $18 \mathrm{H} 10,20 \mathrm{~J} 05$.

Key words and phrases. Homology in varieties of groups.

Copyright (ㅇ 1972, American Mathematical Society 
$\phi_{n}(\mathfrak{W}, \mathfrak{B}, \Pi, A)$ is the "change of variety morphism"; some or all of $\mathfrak{B}, \mathfrak{B}, \Pi$ and $A$ will generally be omitted from the notation.

The following results are routine; we omit the proofs.

LEMMA 1.1. $\phi_{0}: \operatorname{Diff}(\Pi, A) \rightarrow \operatorname{Diff}(\Pi, A)$ is the identity map.

We shall see later that $\phi_{1}$ is a surjection.

LEMMA 1.2. If $\mathfrak{X}$ is a variety containing $\mathfrak{W}$,

$$
\phi_{*}(\mathfrak{X}, \mathfrak{B})=\phi_{*}(\mathfrak{X}, \mathfrak{W}) \phi_{*}(\mathfrak{W}, \mathfrak{B}) \text {. }
$$

Lemma 1.3. $\phi_{*}(\Gamma, A)$ is natural in $\Gamma \rightarrow \Pi \in|(\mathfrak{B}, \Pi)|$ and in left $\mathfrak{B} \Pi$-modules $A$.

LEMMA 1.4. Given a short exact sequence of $\mathfrak{B} \Pi$-modules, $\phi_{*}$ commutes with the appropriate connecting homomorphisms.

LEMMA 1.5. If $\alpha: \Gamma_{0} \rightarrow \Gamma_{1}$ is a surjection in $(\mathfrak{B}, \Pi)$, there is a commutative diagram

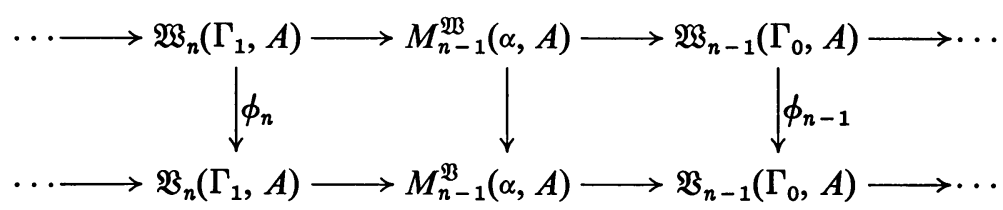

whose rows are Rinehart's exact sequence as in [HI, (2.1)], which was equated with the Barr-Beck exact sequence in [HII, $\$ 1]$.

LEMMA 1.6. There is a commutative diagram

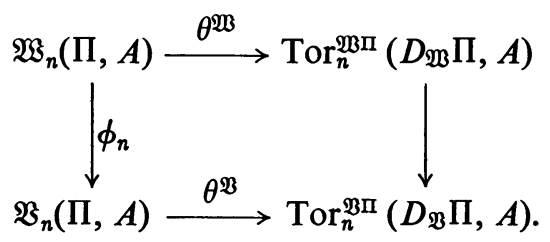

Here $\theta^{\mathfrak{B}}=\theta_{n}^{\mathfrak{P}}(\Pi, A)$ and $\theta^{\mathfrak{W}}=\theta_{n}^{\mathfrak{M}}(\Pi, A)$ as in [HII, §1], and $\operatorname{Tor}_{n}^{\mathfrak{M} \Pi}\left(D_{\mathfrak{B}} \Pi, A\right)$ $\rightarrow \operatorname{Tor}_{n}^{\mathfrak{B} \Pi}\left(D_{\mathfrak{B}} \Pi, A\right)$ is the "change of rings" homomorphism given by the unique $\delta$-morphism of $\operatorname{Tor}_{n}^{\mathfrak{M} \Pi}\left(D_{\mathfrak{B}} \Pi,-\right)$ to $\operatorname{Tor}_{n}^{\mathfrak{B} \Pi}\left(D_{\mathfrak{B}} \Pi,-\right)$, regarded as $\delta$-functors from the category of left $\mathfrak{B} \Pi$-modules to Ab, which is the identity in dimension 0.

Dually there are homomorphisms $\phi^{n}(\mathfrak{B}, \mathfrak{W}, \Pi, A): \mathfrak{B}^{n}(\Pi, A) \rightarrow \mathfrak{W}^{n}(\Pi, A)$ (note the change of direction), and Lemmas 1.1 to 1.6 all dualize.

2. A spectral sequence. The spectral sequence which appears below has the same $E^{2}$ terms and limit as can be obtained as a special case of spectral sequences 
due to André [1], Bachmann [2], Rinehart [13], and Ulmer [20]. These are obtained by varying the first or nonabelian category (here the variety). The spectral sequence obtained by varying the second or abelian category was discussed in [HII, §3]. The point of our treatment (which will generalize) is to calculate the edge effects. The object is to connect the homology in $\mathfrak{B}$ with the homology in $\mathfrak{B}$; one edge homomorphism will be the "change of variety" morphism of $\S 1$; we now introduce the other. Recall that if $T:(\mathfrak{B}, \Pi) \rightarrow A b$ is any functor, the derived functors $\mathfrak{B}_{n}(\Pi, T)$ have been defined as in [HI, §2], $\mathfrak{B}_{n}(\Pi, A)$ being an abbreviation for $\mathfrak{B}_{n}(\Pi, \operatorname{Diff}(-, A))$. $T$ need only be defined on the full subcategory of $\mathfrak{B}$-free groups (over $\Pi$ ), in which case $\mathfrak{B}_{0}(\Pi, T)$ is the Kan extension of $T$ evaluated at $\Pi$; however we shall assume that $T$ is defined on $(\mathfrak{B}, \Pi)$. In this case there is a homomorphism $\lambda: \mathfrak{B}_{0}(\Pi, T) \rightarrow \Pi T$ defined by various authors. For example the right exact functors from $(\mathfrak{B}, \Pi)$ to $\boldsymbol{A} \boldsymbol{b}$ in the sense of Rinehart [13] form a reflective subcategory of $\boldsymbol{A} \boldsymbol{b}^{(\mathfrak{B}, \Pi)}$ (pace set theorists) in the sense of Mitchell [26], and $\lambda$ is the reflection (evaluated at $T$ and $\Pi$ ), cf. [13, p. 299]. Alternatively, if $P_{*} \stackrel{\varepsilon}{\rightarrow} \Pi$ is a simplicial resolution of $\Pi$ by $\mathfrak{B}$-splitting groups, then $\lambda$ is the unique homomorphism to make

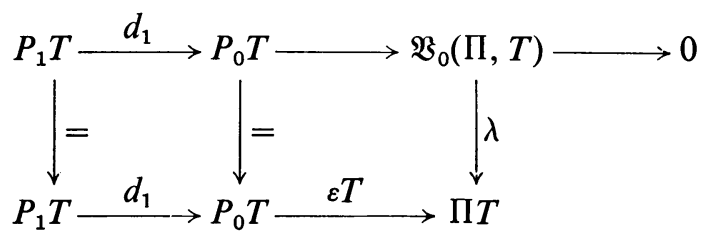

commute. Here $d_{1}=\delta_{1}^{0} T-\delta_{1}^{1} T$ as in [HI, §2]; the top row is exact by definition.

THEOREM 2.1. There is a spectral sequence

$$
\mathfrak{B}_{p}\left(\Pi, \Lambda \cdot \mathfrak{B}_{q}(-, A)\right) \underset{p}{\Rightarrow} \mathfrak{W}_{n}(\Pi, A)
$$

where $\Lambda:(\mathfrak{B}, \Pi) \rightarrow(\mathfrak{B}, \Pi)$ is the inclusion functor, whose edge effects are given by:

$$
\mathfrak{W}_{n}(\Pi, A) \rightarrow E_{n 0}^{2}=\mathfrak{B}_{n}\left(\Pi, \Lambda \cdot \mathfrak{B}_{0}(-, A)\right)=\mathfrak{B}_{n}(\Pi, A)
$$

is the "change of variety" morphism $\phi_{n}$, and

$$
E_{0 n}^{2}=\mathfrak{B}_{0}\left(\Pi, \Lambda \cdot \mathfrak{W}_{n}(-, A)\right) \rightarrow \mathfrak{B}_{n}(\Pi, A)
$$

is the homomorphism $\lambda$ above applied to the functor $\Lambda \cdot \mathfrak{W}_{n}(-, A)$.

Proof. We first adjust the notation. If $T:(\mathfrak{B}, \Pi) \rightarrow A b$ is a functor, the complex of abelian groups from which the derived functors $\mathfrak{B}_{n}(\Pi, T)$ are calculated from the Barr-Beck resolution will be written as $B_{*}^{\mathfrak{Q}}(\Pi, T)$, unless $T=\operatorname{Diff}(-, A)$ which will be abbreviated to $A$. The augmentation $B_{0}^{\mathfrak{B}} \Pi \rightarrow \Pi$ will be $\varepsilon^{\mathfrak{B}}$.

Form the first quadrant double complex $T_{p q}=B_{p}^{\mathfrak{g}}\left(\Pi, \Lambda \cdot B_{q}^{\mathfrak{Q}}(-, A)\right.$ ), where $\Lambda:(\mathfrak{B}, \Pi) \rightarrow(\mathfrak{B}, \Pi)$ is the inclusion functor. Fixing $p$ and taking homology gives $B_{p}^{\mathfrak{B}}\left(\Pi, \Lambda \cdot \mathfrak{B}_{q}(-, A)\right)$, and taking homology again gives $\mathfrak{B}_{p}\left(\Pi, \Lambda \cdot \mathfrak{B}_{q}(-, A)\right)$. Fixing $q$ and taking homology in $T$ gives $\mathfrak{B}_{p}\left(\Pi, \Lambda \cdot B_{q}^{\mathfrak{P}}(-, A)\right)$. Now $\Lambda \cdot B_{q}^{\mathfrak{P}}(-, A)$ 
factors through the comma category of sets over the underlying set of $\Pi$ and hence is flask in the sense of Rinehart [13] (cf. Rinehart [14] and the proof of [HII, Proposition 1.1]). Thus $\mathfrak{B}_{p}\left(\Pi, \Lambda \cdot B_{q}^{\mathfrak{Q}}(-, A)\right)=0$ for $p>0$ and

$$
\mathfrak{B}_{0}\left(\Pi, \Lambda \cdot B_{q}^{\mathfrak{M}}(-, A)\right)=B_{q}^{\mathfrak{M}}(\Pi, A) .
$$

Taking homology again gives $\mathfrak{W}_{q}(\Pi, A)$, and so the first part of the theorem is proved. We calculate the edge effects using the same techniques as in the proof of [HII, Theorem 3.1]. Recall [22, Theorem XI, 4.4] which states that if $I_{p q}^{r}$ is the first spectral sequence of the first quadrant double complex $S_{p q}$ then the edge effects are given by $I_{0 n}^{1}=H_{n} S_{0 *} \rightarrow H_{n} S$ induced by the inclusion of $S_{0 *}$ in $A$, and $H_{n} S \rightarrow H_{n}(S / M)=I_{n 0}^{2}$ induced by the projection of $S$ on $S / M$, where $\boldsymbol{M}$ is the subcomplex of $\boldsymbol{S}$ given by

$$
M_{n}=\sum_{p+q=n ; q>0} S_{p q} \cup \partial \sum_{p+q=n+1 ; q>0} S_{p q} .
$$

We apply this first to the second spectral sequence of $\boldsymbol{T}$ (which collapses), that is with $\boldsymbol{S}=\boldsymbol{T}$ transposed. In this case $\boldsymbol{S} / \boldsymbol{M}$ is chain isomorphic to $B_{*}^{\mathfrak{M}}(\Pi, A)$ via $B_{*}^{\mathfrak{M}}\left(\varepsilon^{\mathfrak{B}}, A\right)$. Hence we have an isomorphism $\omega$ of $H_{*} T$ onto $\mathfrak{W}_{*}(\Pi, A)$. Now looking at the first spectral sequence and applying the first part of the above theorem gives us the homomorphism $\zeta$ in the diagram

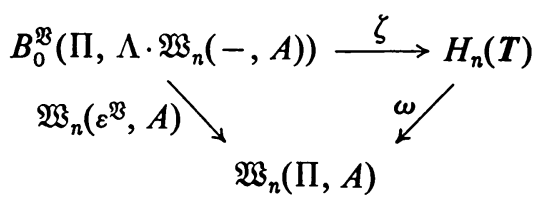

which is clearly commutative. This identifies the edge homomorphism $E_{0 n}^{1} \rightarrow H_{n}(T)$ as $\mathfrak{W}_{n}\left(\varepsilon^{\mathfrak{B}}, A\right)$ "up to $\omega$ ". It follows from the definition of $\lambda$ above that $\lambda$ is the edge homomorphism $E_{0 n}^{2} \rightarrow H_{n}(\boldsymbol{T})$, again "up to $\omega$ ". We now turn to the base. Define the first quadrant double complex $\bar{T}$ by $\bar{T}_{p q}=B_{p}^{\mathfrak{B}}\left(\Pi, B_{q}^{\mathfrak{B}}(-, A)\right)$. Dividing by the verbal subgroup defined by $\mathfrak{B}$ induces a functor of $(\mathfrak{W}, \Pi)$ into $(\mathfrak{B}, \Pi)$ and hence a chain map $F: T \rightarrow \bar{T}$. Now let $\boldsymbol{M}_{1}, \boldsymbol{M}_{2}, \bar{M}_{1}$ and $\bar{M}_{2}$ correspond to $\boldsymbol{M}$ in the theorem quoted above, where $\boldsymbol{S}$ is taken as $\boldsymbol{T}, \boldsymbol{T}$ transposed, $\overline{\boldsymbol{T}}$ and $\overline{\boldsymbol{T}}$ transposed respectively. Then $\boldsymbol{T} / \boldsymbol{M}_{1}, \overline{\boldsymbol{T}} / \overline{\boldsymbol{M}}_{1}$, and $\bar{T} / \bar{M}_{2}$ are naturally isomorphic to $B_{*}^{\mathfrak{B}}(\Pi, A)$, and $\boldsymbol{T} / \boldsymbol{M}_{2}$ is isomorphic to $B_{*}^{\mathfrak{M}}(\Pi, A)$. Using these identifications, $F$ induces a commutative diagram

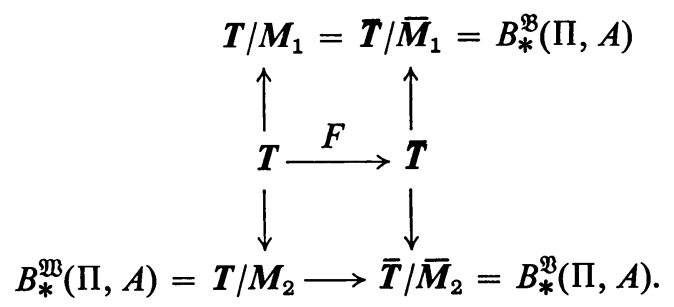


Taking homology now gives

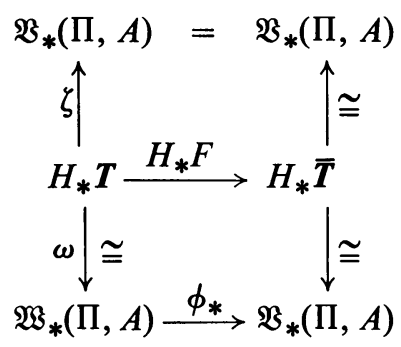

where $\omega$ is the isomorphism of the first part and $\zeta$ is the base homomorphism. It only remains to prove that the composite of either homomorphism on the East side with the inverse of the other is the identity. Now $H_{*} \bar{T}$ and $\mathfrak{B}_{*}(\Pi,-)$ may clearly be regarded as $\delta$-functors from the category of functors from $\mathfrak{B}$-free groups over $\Pi$ to $\boldsymbol{A b}$, and as such they are effaced on the left by the projective functors. Moreover, since we are dealing with $\delta$-functors, it is enough to look at dimension zero. But in this case the result may be read off at once by looking at $\overline{\boldsymbol{T}}$ with its canonical augmentation.

COROLlaRY 2.2. There is an exact sequence

$$
\begin{aligned}
& \mathfrak{W}_{2}(\Pi, A) \stackrel{\phi_{2}}{\longrightarrow} \mathfrak{B}_{2}(\Pi, A) \longrightarrow \mathfrak{B}_{0}\left(\Pi, \Lambda \cdot \mathfrak{W}_{1}(-, A)\right) \\
& \stackrel{\lambda}{\longrightarrow} \mathfrak{W}_{1}(\Pi, A) \stackrel{\phi_{1}}{\longrightarrow} \mathfrak{B}_{1}(\Pi, A) \longrightarrow 0 .
\end{aligned}
$$

In particular, $\phi_{1}$ is a surjection. Of course the results of this paragraph all dualize. By a well-known folk theorem (but see Beck [5]), $\mathfrak{B}^{1}(\Pi, A)$ classifies the extensions of $A$ by $\Pi$ that lie in $\mathfrak{B}$, and it can be shown that with this identification the injection $\phi^{1}: \mathfrak{B}^{1}(\Pi, A) \rightarrow \mathfrak{W}^{1}(\Pi, A)$ is the inclusion of the set of extensions of $A$ by $\Pi$ that lie in $\mathfrak{B}$ in the set of those that lie in $\mathfrak{B}$.

3. The second homology groups. The striking behaviour of $\mathfrak{B}_{2}(\Pi, A)$ is illustrated, using (2.1), when $\Pi$ is a finitely generated abelian group and $A=Z$.

The abelian group $\Pi$ is of type $\left(s ; n_{1}, \ldots, n_{t}\right)$ if the torsion subgroup $T$ of $\Pi$ is of direct product of cyclic subgroups of order $n_{1}, \ldots, n_{t} ; n_{1}>1, n_{i} \mid n_{i+1}$ for $i=1, \ldots$, $t-1$; and $\Pi / T$ is of rank $s . s+t$ is the rank of $\Pi$. In quoting (2.1) the symbol $\Lambda$ will be omitted. Define a function $\gamma$ of two positive integers by

$$
\gamma(r, c)=\frac{1}{c+1} \sum_{d \mid(c+1)} \mu(d) r^{(c+1) / d}
$$

Here $\mu$ is the Möbius function; if $n$ is the product of $u$ distinct primes $(u \geqq 0)$, then $\mu(n)=(-1)^{u}$; else $\mu(n)=0$.

LEMMA 3.1. If $\Pi$ is a finitely generated abelian group of rank $r>0$ and type $\left(s ; n_{1}, \ldots, n_{t}\right)$, and $\mathfrak{B}=\mathfrak{N}_{c}$, then $\mathfrak{B}_{0}\left(\Pi, H_{2}(-, Z)\right)$ is of rank $\rho$ and type 
$\left(\sigma ; \nu_{1}, \ldots, \nu_{\tau}\right)$ where $\rho=\gamma(r, c), \sigma=\gamma(s, c)$, and $\nu_{\tau}=n_{t}$ if $s>0, \nu_{\tau}=n_{t-1}$ if $s=0$. (In particular, if $r=s, \rho=\sigma$ and if $r=1, \rho=0$.)

Proof. Let

$$
\Pi=C\left(a_{1}\right) \times \cdots \times C\left(a_{s}\right) \times C_{n_{1}}\left(a_{s+1}\right) \times \cdots \times C_{n_{t}}\left(a_{r}\right)
$$

with the obvious notation, let $F$ be $\mathfrak{R}_{c}$-freely generated by $x_{1}, \ldots, x_{r}$, and define $f: F \rightarrow \Pi$ by $x_{i} f=a_{i}, i=1, \ldots, r$. The fibre product $F \times_{\Pi} F$ is the subgroup of $F \times F$ consisting of elements $(p, q)$ such that $p f=q f$, and $(p, q) \mapsto\left(p q^{-1}, q\right)$ is an isomorphism of $F \times_{\Pi} F$ onto the split extension $R F$ of $R$ by $F$, where $R$ is the kernel of $f . R$ is generated qua subgroup by $x^{n_{i-s}}, i=s+1, \ldots, r$, and $w_{1}, \ldots, w_{k}$, say, where $w_{i}$ is a commutator for all $i$. Defining a homomorphism of a group $G$ into $F \times \times_{\mathrm{\Pi I}} F$ is equivalent to defining a homomorphism $\left(g_{1}, g_{2}\right)$ of $G$ into $F \times F\left(g_{i}: G \rightarrow F\right)$ such that $g_{1} f=g_{2} f$. Let $\bar{F}$ be $\mathfrak{R}_{c}$-freely generated by $y_{1}, \ldots, y_{r}, z_{s+1}, \ldots, z_{r}$, $v_{1}, \ldots, v_{k}$, and define $\left(g_{1}, g_{2}\right): \bar{F} \rightarrow F \times_{\Pi} F$ by $y_{i} g_{1}=x_{i}, z_{i} g_{1}=x^{n_{i-s}}, v_{i} g_{1}=w_{i}, y_{i} g_{2}$ $=x_{i}, z_{i} g_{2}=1, v_{i} g_{2}=1$. Then $\left(g_{1}, g_{2}\right)$ is a surjection, and by [13, p. 299], $\mathfrak{B}_{0}\left(\Pi, H_{2}(-, Z)\right)$ is the cokernel of $H_{2}\left(g_{1}, Z\right)-H_{2}\left(g_{2}, Z\right): H_{2}(\bar{F}, Z) \rightarrow H_{2}(F, Z)$. It is easy to see that the Schur multiplier of the $\mathfrak{N}_{c}$-free group on a set $\mathfrak{x}$ is the $(c+1)$ th lower central factor of the absolutely free group on $\mathfrak{x}$; that is, the free abelian group on the basic commutators of weight $c+1$ in $\mathfrak{x}$; these multipliers will be written additively. One sees easily that $H_{2}\left(g_{1}, Z\right)-H_{2}\left(g_{2}, Z\right)$ has the following properties:

(i) the image of a basic commutator (of weight $c+1$ ) in $\bar{F}$ is a multiple of a basic commutator in $F$ or 0 ;

(ii) if $\left[x_{i_{1}}, \ldots, x_{v_{c}+1}\right]$ is a basic commutator in $F$ (not necessarily left normed), and if $\max \left(i_{1}, \ldots, i_{c+1}\right) \leqq s$, then no nonzero multiple of $\left[x_{i_{1}}, \ldots, x_{i_{c+1}}\right]$ is the image of a basic commutator, whereas

(iii) if $i_{\alpha}>s$ for some $\alpha$ and $i_{\alpha}$ is the least such integer, then $n_{i_{\alpha}-s}\left[x_{i_{1}}, \ldots, x_{i_{c+1}}\right]$ is the image of a basic commutator, and if $m\left[x_{i_{1}}, \ldots, x_{i_{c}+1}\right]$ is such an image, then $n_{i_{\alpha}-s} \mid m$. Note that if $s>0, i_{\alpha}=r$ for any basic commutator involving $x_{1}$ and $x_{r}$ only; whereas if $s=0, i_{\alpha} \leqq r-1$ and $i_{\alpha}=r-1$ for any basic commutator involving $x_{r-1}$ and $x_{r}$ only.

Of course neither (ii) nor (iii) occurs if $r=1$ in which case $H_{2}(F, Z)$ is trivial, and (iii) only occurs if $r>s$, so degenerate cases give no trouble. Finally the number of basic commutators of weight $c+1$ on $r$ letters is $\gamma(r, c)$ (see [9]). Putting all this together gives the lemma.

It is now easy to prove the following

THEOREM 3.2. If $\Pi$ is a finitely generated abelian group of rank $r>0$ and type $\left(s ; n_{1}, \ldots, n_{t}\right)$, and $\mathfrak{B}=\mathfrak{N}_{c}$, then $\mathfrak{B}_{2}(\Pi, Z)$ is of rank $\rho$ and type $\left(\sigma ; \nu_{1}, \ldots, \nu_{\tau}\right)$ where

$0 \leqq \rho-\gamma(r, c) \leqq t+r(r-1)(r-2) / 6$ (cf. (3.1)),

$0 \leqq \sigma-\gamma(s, c) \leqq s(s-1)(s-2) / 6$,

$\nu_{\tau}$ divides $n_{t}^{2}$, and if $s=0, \nu_{\tau}$ divides $n_{t-1} n_{t}$.

In particular, as $c \rightarrow \infty, \rho \sim r^{c+1} /(c+1)$ and $\sigma \sim s^{c+1} /(c+1)$. 
Proof. Apply Corollary 2.2 with $\mathfrak{W}$ the universal variety and $A=Z$. By [HII, Proposition 2.3], $\mathfrak{W}_{1}(\Pi, Z)$ and $\mathfrak{B}_{1}(\Pi, Z)$ are isomorphic; and being Hopf groups, $\phi_{1}$ is an isomorphism. (In fact $\phi_{1}$ and $\theta_{1}^{\mathfrak{B}}(\Pi, Z)$ are inverses, see Lemma 1.6.) Since $\mathfrak{W}_{2}(\Pi, Z)=H_{3}(\Pi, Z)$ a routine calculation gives

$$
\mathfrak{W}_{2}(\Pi, Z)=Z^{s(s-1)(s-2) / 6} \oplus \bigoplus_{i=1}^{t} Z_{n_{\mathfrak{i}}}^{1+(r-i)(r-i-1) / 2}
$$

where $Z_{m}$ denotes $Z / m Z$, and $A^{k}$ denotes the direct sum of $k$ copies of $A$. So $\mathfrak{W}_{2}(\Pi, Z)$ is of rank $r(r-1)(r-2) / 6+t$ and type $\left(s(s-1)(s-2) / 6 ; n_{1}, n_{1}, \ldots, n_{t}, n_{t}\right)$. Now applying Lemma 3.1 gives the result.

In particular, the homology in dimension 2 of a product is not related in a simple way to the homology of the factors, whereas in dimension 1 there is a Künneth formula of sorts (cf. [HI, Theorem 5.2]).

To obtain results in cohomology, apply universal coefficients [HI, Lemma 4.1]. If $\Pi$ is finite, $\mathfrak{B}^{2}(\Pi, Q / Z) \cong \mathfrak{B}_{2}(\Pi, Z)$. The wild behaviour of this group if $\mathfrak{B}=\mathfrak{R}_{c}$ can hardly be reflected in an obstruction theory.

\section{BIBLIOGRAPHY}

25. C. R. Leedham-Green, Homology in varieties of groups. II, Trans. Amer. Math. Soc. 162 (1971), 15-25.

26. B. Mitchell, Theory of categories, Pure and Appl. Math., vol. 17, Academic Press, New York, 1965. MR 34 \#2647.

Queen Mary College, London, England 